\title{
O conhecimento do enfermeiro sobre a prevenção do câncer de colo de útero na atenção básica*
}

\section{The nurse's knowledge nurse cervical cancer prevention in primary care}

\author{
José Paulo Santos Conceição' • Mariane Mattos da Silva Medeiros² • Lilia Marques Simões Rodrigues ${ }^{3}$ •
} Márcia Ribeiro Bráz ${ }^{4}$ Carlos Marcelo Balbino ${ }^{5}$ Zenith Rosa Silvino 6

\begin{abstract}
RESUMO
Objetiva-se identificar o conhecimento do enfermeiro da Atenção Básica quanto à prevenção do câncer de colo de útero, descrever a percepção do enfermeiro quanto à prevenção deste câncer,e identificar as atividades de prevenção realizadas pela Atenção Básica de Saúde.Constitui-se uma pesquisa de natureza exploratória com abordagem qualitativa, com 15 enfermeiras atuantes nas unidades de Atenção Primária da cidade de Valença, Rio de Janeiro, durante outubro de 2014, com entrevistas estruturadas com os profissionais. Foram discutidas cinco categorias sobre prevenção, educação em saúde, coleta do exame citopatológico, vacina contra HPV e ações do planejamento da prevenção do câncer de colo de útero. Conclui-se que as enfermeiras das unidades de atenção primária possuem certo conhecimento sobre as medidas de prevenção preconizadas pelo Ministério da Saúde, e algumas destas são realizadas nas unidades, porém com determinadas fragilidades do sistema.
\end{abstract}

Palavras-chave: Atenção Básica; Neoplasia do Colo do Útero; Prevenção de Doenças; Enfermagem.

\begin{abstract}
The aim is to identify nurse's knowledge of basic care regarding the prevention of cervical cancer, to describe the nurses' perception regarding the prevention of this cancer, and to identify the prevention activities performed by Primary Health Care. An exploratory research with a qualitative approach, with 15 working nurses in the Primary Care Unit of the Valença city, Rio de Janeiro, duringoctober 2014, with structured interviews with the professionals. Five categories on prevention, health education, cytopathological examination, HPV vaccine, and cervical cancer prevention planning actions were discussed. It concludes that nurses in the primary care units have some knowledge about the preventive measures recommended by the Ministry of Health, and some of these are carried out in the units, but with certain weaknesses of the system.
\end{abstract}

Keywords: Primary Health Care; Uterine Cervical Neoplasms; Disease Prevention; Nursing.

*Trabalho realizado no Centro de ensino Superior de Valença (CESVA). Valença (RJ), Brasil.

${ }^{1}$ Enfermeiro. Egresso do Curso de Enfermagem do CESVA. Valença (RJ), Brasil.

${ }^{2}$ Enfermeira. Egressa do Curso de Enfermagem do CESVA. Valença (RJ), Brasil.

${ }^{3}$ Enfermeira . Mestre em Enfermagem Assistencial /UFF. Professora e Coordenadora do Curso de Enfermagem da USS. Vassouras (RJ), Brasil.

${ }^{4}$ Enfermeira. Doutora em Enfermagem /UFRJ. Professora e Coordenadora do Curso de Enfermagem da UNIG. Pinheiral (RJ), Brasil.

${ }^{5}$ Enfermeiro. Doutorando do Programa do Programa em Ciências do Cuidado em Saúde pela Universidade Federal Fluminense - UFF. Mestre em Enfermagem pela Universidade Federal Fluminense/MPEA/UFF. Professor da Universidade Severino Sombra - USS e Centro de Ensino Superior de Valença CESVA/FAA - Faculdade de Enfermagem de Valença. Rua 206 número 34, Conforto. Volta Redonda (RJ), Brasil. Telefone: 24 - 999183696. E-mail: carlosmbalbino@hotmail.com. Autor correspondente.

${ }^{6}$ Enfermeira, Pós Doutora e Doutora em Enfermagem,Professora da Universidade Federal Fluminense - UFF, Niterói (RJ). 


\section{INTRODUÇÃO}

O câncer de colo de útero se desenvolve a partir de alterações no colo do útero, que se localiza no fundo da vagina. Tais lesões, chamadas de lesões precursoras, são curáveis na maioria das vezes e podem demorar anos para que se modifiquem e se tornem células cancerígenas, se não tratadas ${ }^{1}$.

O câncer do colo do útero ocupa a quarta posição dos tipos mais comuns de câncer entre as mulheres, e é responsável pelo óbito de 265 mil mulheres por ano. Esta neoplasia representou 5.160 óbitos, uma taxa de mortalidade ajustada para a população mundial de 4,66 óbitos para cada 100 mil mulheres².

O câncer de colo uterino representa um problema de saúde pública no Brasil, por ser uma doença de evolução lenta e por exercer um impacto importante nas altas taxas de prevalência e na morbimortalidade em mulheres na fase produtiva de suas vidas ${ }^{3}$.

A atenção básica orienta-se pelos princípios do Sistema único de Saúde ${ }^{1}$ da universalidade e integralidade da atenção. Nesta compreensão, para a efetiva implementação das ações em saúde, deve-se investir em estratégias que proporcionem a construção de vínculo, a continuidade do cuidado e a humanização da assistência.

No Estado do Rio de Janeiro, ainda nos anos de 2014 e 2015, as estimativas de incidência para o período e localidade são de: 8.380 novos casos de câncer de mama; 2.580 novos casos de câncer de cólon e reto; 1.340 novos casos de câncer de útero e 1.240 novos casos de câncer de traqueia, brônquios e pulmão².

A evolução das técnicas oncológicas tem sido fundamental para a sobrevida do paciente oncológico, porém as estatísticas de câncer tendem a aumentar, caso abranjam medidas de prevenção que não sejam implantadas ${ }^{2}$. Ainda sobre as técnicas oncológicas, compreendem que o medo do diagnóstico e o receio pela escolha do tipo de tratamento, por gerar efeitos adversos, podem ser um fator agravante para a resistência à busca pelo serviço de saúde e resulta em diagnóstico em tempo prejudicial ao bom prognóstico ${ }^{4}$.

O diagnóstico do câncer de colo de útero é feito por meio do exame citopatológico (Papanicolau), preferencialmente pelas mulheres que têm ou já tiveram atividade sexual. Os exames devem ser feitos com intervalo de um ano e, se os resultados forem normais, o exame será realizado a cada três anos. Tal tipo de câncer, em estágios iniciais, é assintomático, o que sugere que se não diagnosticado nesta fase, há a invasão do colo uterino e dos tecidos adjacentes, podendo apresentar sangramento durante a relação sexual e dispareunia 5 .

No desenvolvimento do câncer de colo de útero especificamente, o principal fator de risco está relacionado à infecção causada pelo Papilomavírus Humano (HPV). Os principais fatores de risco relacionados ao desenvolvimento de infecção pelo HPV são: início precoce das atividades sexuais; multiplicidade de parceiros sexuais; parceiro sexual masculino com múltiplas parceiras; tabagismo; infecções genitais de repetição (não somente as viróticas) ${ }^{6}$.

Devido ao alto índice de novos casos do câncer de colo de útero, em 2014 é instituída a campanha de vacinação contra o vírus do HPV em meninas de 11 a 13 anos, buscando prevenir a infecção pelo vírus do HPV. Esta vacina é a quadrivalente, que previne contra os seguintes tipos de HPV: $6,11,16$ e 18 , sendo os tipos 16 e 18 os correspondentes por $70 \%$ dos casos de câncer de colo de útero, responsáveis atualmente por $95 \%$ dos casos de câncer no país. Há mais de 100 tipos de HPV e o vírus pode infectar através da pele ou mucosas. Pelo menos 13 tipos do vírus têm potencial para causar câncer ${ }^{7}$.

O exame citopatológico preventivo do câncer do colo de útero é a principal estratégia para detecção precoce da doença no Brasil.A igualdade de oportunidades em relação à promoção da saúde garante às pessoas a realização de seus potenciais de saúde. A promoção da saúde abrange as atitudes e habilidades em todas as etapas da vida, devendo acontecer nos mais variados espaços coletivos. Para que a promoção da saúde ocorra, é necessário que ela esteja vinculada ao processo educativo, destacando os fatores de risco modificáveis, como hábitos de risco, entre outros ${ }^{8}$.

0 enfermeiro é imperativo na equipe de saúde para a prevenção do câncer de colo uterino ${ }^{9}$. Por estar próximo da população, o enfermeiro tem papel fundamental na criação do vínculo com a comunidade, com a educação em saúde desenvolvida nas comunidades e escolas. Além de realizar o exame citopatológico e dar destaque na prevenção esclarecendo dúvidas das usuárias, realizando busca ativa das mulheres faltosas ${ }^{10}$. Para isso, é necessário que haja por parte do enfermeiro, conhecimento acerca dos tratamentos, dos avanços da oncologia, mesmo que esteja inserido na Atenção Básica ${ }^{11}$, pois trata-se de uma área, na qual a enfermagem atua ativamente nas medidas preventivas e na educação em saúde, o que permite sua atuação como integrante essencial na equipe multiprofissional de saúde ${ }^{1}$.

0 objeto do presente estudo é a enfermagem frente à prevenção do câncer de colo de útero na Atenção Básica de Saúde.

Neste sentido, surgem as seguintes questões norteadoras: qual a percepção do enfermeiro que atua na Atenção Básica de Saúde quanto à prevenção do câncer de colo de útero? Quais as medidas de prevenção instituídas pelo Ministério da Saúde na prevenção do câncer do colo do útero? A partir destas questões, chegamos aos objetivos: descrever a percepção do enfermeiro quanto à prevenção do câncer de colo de útero e analisar as atividades de prevenção que são realizadas pelos enfermeiros da Atenção Básica de Saúde.

\section{MÉTODO}

Pesquisa descritiva e exploratória, com abordagem qualitativa ${ }^{12}$. 
O cenário da pesquisa foi a Estratégia Saúde da Família (ESF) e Unidades Básicas de Saúde (UBS) do município de Valença, Estado do Rio de Janeiro, com uma área 1.304,813 $\mathrm{Km}^{2}$, com 71.843 habitantes ${ }^{13}$. O município é composto pelos seguintes distritos: Conservatória, Santa Isabel, Pentagna, Parapeúna e Barão de Juparanã, tendo Valença como sede do município.

Foram sujeitos do estudo todos os 15 enfermeiros que atuam na Rede Básica, que é composta por 13 unidades da ESF, 01 unidade com 02 equipes, e 01 sem enfermeiro, totalizando 13 enfermeiros, e 08 UBS, distribuídos 02 enfermeiros para as 08 unidades. Foram selecionados seguindo o critério de inclusão: atuar na Atenção Básica de Saúde e concordar em participar da pesquisa. Essa amostra corresponde a $100 \%$ dos enfermeiros que atuam na Rede Básica de Saúde do município de Valença.

Para a geração de dados, utilizamos a técnica de entrevista estruturada, na qual criamos um roteiro de entrevista, para facilitar na coleta de dados.

As entrevistas ocorreram em outubro de 2014, sendo gravadas em aparelho de multimídia do tipo MP4, após consentimento dos entrevistados. Antes de iniciar a entrevista os participantes receberam explanação do que se trata a pesquisa e assinaram o Termo de Consentimento e Livre Esclarecido.

Para a análise dos dados foi utilizada a análise de conteúdo, que pressupõe algumas etapas como: pré-analise; exploração do material ou codificação, e tratamento dos resultados (inferência e interpretação) ${ }^{14}$.

Atendendo às exigências da Resolução 466/12, do Conselho Nacional de Saúde, o projeto de pesquisa foi analisado e aprovado com o número 791.720 pelo Comitê de Ética Faculdade de Medicina de Valença.

Para preservar o anonimato dos participantes, na apresentação dos resultados, os enfermeiros foram nomeados com a letra "E" e o número arábico sequencial.

\section{RESULTADOS E DISCUSSÃO}

$O$ estudo foi realizado com enfermeiros que atuam na Atenção Básica de saúde tanto nas ESF, quanto nas UBS na cidade de Valença, e avaliou-se o conhecimento do enfermeiro sobre a prevenção do câncer de colo de útero na Atenção Básica. Durante todo o período de geração de dados houve interesse dos sujeitos participantes em contribuir no sentido de aprimorar as estratégias de prevenção do câncer de colo de útero para população feminina do município.

0 estudo foi realizado com 15 enfermeiras. Quanto sua atuação na Atenção Básica de Saúde, 13 atuam na ESF e 02 na UBS. A faixa etária predominante é de 21 a 40 anos, na qual 11 enfermeiras estão nesta faixa e apenas $02 \mathrm{com}$ mais de 40 anos. Uma delas preferiu não identificar a idade.

Com relação à formação acadêmica, 07 enfermeiras possuem de 1 a 5 anos de conclusão da graduação, 04 enfermeiras possuem de 6 a 10 anos, e 04 enfermeiras possuem de 11 a 15 anos.

$E$, no que concerne à experiência profissional, atuando como enfermeiro da Atenção Básica, 01 enfermeira atua há 07 meses, 07 enfermeiras atuam de 1 a 5 anos, e 03 atuam de 11 a 15 anos.

Quanto à possuir pós-graduação no nível de especialização: 03 referiram possuir pós-graduação, 04 com mais de uma e 02 não possuem, sendo que, 09 em Saúde da Família, 02 em Gestão Pública, 02 em Enfermagem do Trabalho, 01 em CTI, 01 em Cardiologia e Hemodinâmica e 02 enfermeiras não especificaram qual pós-graduação possuíam.

O material submetido à analise de conteúdo foi interpretado, discutido e originou as seguintes categorias: 1. Conhecimento de prevenção como fator relevante; 2 . Educação em saúde como atividade de prevenção; 3. Coleta de preventivo como ação base; 4. Inclusão da vacina do HPV e, 5. Ações consideradas no planejamento da prevenção.

As enfermeiras entrevistados demonstraram interesse na realização das tarefas voltadas para a prevenção do câncer de colo de útero em todas as unidades de Atenção Básica de Valença, além de possuírem o conhecimento elementar para conduzir as práticas de prevenção. Mesmo com as dificuldades de gestão, como a falta de materiais no município, são realizadas tarefas de prevenção como educação em saúde e vacinação contra o vírus HPV nas escolas, e ainda há as atividades preventivas e sua parcela de efetividade.

As enfermeiras também relatam que apenas nas escolas e igrejas conseguem atingir um público maior de adolescentes e mulheres para a realização da educação em saúde, quando essas atividades são realizadas nas unidades, apenas um pequeno número de mulheres participa, normalmente entre dois a três participantes, e que a população em sua maioria é carente de informação.

Todos os enfermeiros entrevistados apontam o exame citopatológico, como ação base para a prevenção, além da distribuição de preservativos, porém as unidades não estão realizando esses exames por falta de materiais. É importante destacar que estas ações voltadas para prevenção, além de serem muito benéficas para as mulheres, também são de enorme benefício para os cofres públicos, pois ações de prevenção custam bem mais barato do que um tratamento de uma pessoa com diagnóstico de câncer ${ }^{1}$.

A conscientização é o primeiro passo, para qualquer tipo de prevenção, e o enfermeiro é o grande responsável por esta etapa, na detecção precoce de qualquer patologia,já que a busca por livre demanda das mulheres não é satisfatória para haver a cobertura efetiva das ações de prevenção.

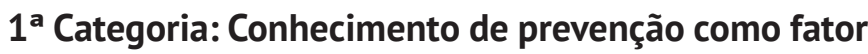 relevante}

O enfermeiro deve estar capacitado com conhecimento bem amplo para trabalhar na prevenção, pois existem 
várias adversidades que podem influenciar, como religião, fator econômico, escolaridade, idade, entre outros 9 . Por isso é tão importante atualizar seus conhecimentos para aplicar cuidado amplo e específico para cada usuária.

Os pontos que mais se destacaram foram: estratégias que utilizem a prevenção como fator no planejamento das ações que diminuem o aparecimento de novos casos de câncer de colo útero.

“Prevenção é pra que não deixe chegar à doença, é quando tem ausência de doença faz educação permanente." (E2)

"Prevenção é orientar pra que não haja agravos na saúde da comunidade, e pode ser através de palestras, grupos de apoio de prevenção das principais doenças. É uma forma de evitar que a doença ocorra." (E3)

"Prevenção são as ações feitas com o objetivo de evitar que apareçam doenças." (E4)

"Prevenir nada mais é do que propor medidas e realizar medidas para que determinada doença não aconteça." (E14)

A segurança no momento da tomada de decisão é dada ao enfermeiro a partir do conhecimento que ele detém, em relação ao paciente, à equipe, às atividades da unidade, entre outros ${ }^{15}$.

Ressalta-se a importância do preparo do enfermeiro na orientação e oferecimento de cuidados específicos às pacientes com câncer ${ }^{11}$.

A contribuição do enfermeiro na prevenção do câncer de colo uterino destaca-se pela sua participação no controle de fatores de risco, na realização da consulta ginecológica e do exame de Papanicolau, o que difunde a qualidade do atendimento à demanda e efetiva, com o sistema de registro adequado ${ }^{16}$.

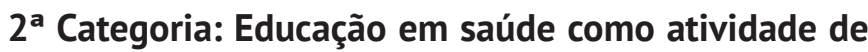 prevenção}

Educação em saúde, sala de espera e palestras foram citadas como medidas de orientação sobre prevenção. A educação em saúde é um dos fatores mais funcionais na prevenção do câncer de útero ${ }^{8}$. Não sabendo as formas de contágio da doença, as formas de tratamento, a importância do uso de preservativo, muitas mulheres deixam de procurar as unidades de saúde, por falta de conhecimento. Por este motivo, é sempre importante realizar educação em saúde em grupos, igrejas, escolas, entre outros.

"As principais (atividades de prevenção) da unidade são a educação contínua e orientação para importância do exame preventivo." (E2)

"Orientações em grupo à respeito do assunto e coleta de preventivo." (E3)

“(O planejamento) Seria a educação em saúde, que automaticamente você faz na sala com o paciente, orientando sobre a importância do exame, ou em conjunto, em grupo." (E7)

"As mulheres aderem mais às atividades de educação em saúde." (E8)

A educação permanente em saúde, as atividades educativas em grupo, as parcerias entre instituições e organizações que trabalhem o tema Câncer de Colo de Útero, que possam encorajar a prevenção da doençå8.

Com a atuação do enfermeiro na educação em saúde, esclarecendo dúvidas e medos das mulheres, o índice de novos casos pode diminuir devido à possibilidade do número de mulheres que se previne, aumentar ${ }^{10}$.

A realização de educação em saúde através do enfermeiro capacitado é fundamental para a prevenção do câncer cérvico-uterino ${ }^{17}$.

\section{$3^{\text {a }}$ Categoria: Coleta de preventivo como ação base}

O exame citopatológico ou Papanicolau ${ }^{1}$, é a forma mais eficiente de detectar precocemente o câncer de colo de útero, e é fundamental a sua realização a partir dos 25 anos de idade. Por isso, as unidades de Atenção Básica devem trazer essas mulheres para a unidade, fazendo busca ativa, estreitando os laços entre a unidade e saúde e paciente.

A coleta do exame citopatológico foi citada em todas as falas das enfermeiras sobre as medidas de prevenção do câncer de colo de útero adotadas pelo Ministério da Saúde e realizadas pelas unidades. Destacam-se: "A principal da unidade é a realização do exame
citopatológico do colo de útero (...)." (E1)

"Exame citpatológico do colo de útero, educação em saúde, sala de espera." (E10)

“Exames preventivos e educação continuada." (E11)

"Nós possuímos o exame Papanicolau, com a faixa etária preconizada pelo Ministério da Saúde (...)."( E15)

A contribuição do enfermeiro intervém no encaminhamento das mulheres com alterações citológicas, além de ter contribuição de grande importância na prevenção do câncer de colo de útero participando no controle dos fatores de risco, realizando consulta ginecológica, atendendo à demanda com qualidade, dentro outros ${ }^{16}$.

O objetivo do Programa de Saúde da Mulher é reduzir a morbimortalidade das mulheres acima de 10 anos de idade, promovendo sua assistência integral. Devido aos altos índices de incidência e mortalidade do câncer de colo de útero e de mama, as ações direcionadas ao rastreamento e ao diagnóstico precoce são realizadas através da coleta do exame citopatológico, do exame clínico de mama e da mamografia, referenciando para análise e tratamento os casos com resultados alterados ${ }^{6}$. 
A principal estratégia de detecção e rastreio do câncer de colo de útero é o exame preventivo do colo de útero, ou exame citopatológico cervico-vaginal e microbiota ${ }^{18}$.

\section{$4^{\text {a }}$ Categoria: Inclusão da vacina do HPV}

O vírus do HPV é um dos principais causadores do câncer de colo de útero. Por isso, a vacina quadrivalente foi incluída no calendário de vacinação contra os subtipos 6 e 11, causadores de verrugas genitais, e 16 e 18, que são os principais responsáveis pelos casos de câncer ${ }^{2}$, sendo fundamental a vacinação das meninas e meninos dentro da faixa etária indicada pelo Ministério da Saúde.

Apenas 03 enfermeiras responderam não ter recebido capacitação sobre a vacina contra HPV, o principal causador do câncer de colo de útero, além de também ter sido citado como medida preventiva essencial do vírus, evitando, consequentemente, as lesões associadas.

"A gente foi capacitada sobre a importância da vacina, como ela age, as contraindicações, os efeitos colaterais, e outros assuntos relacionados à vacina." (E3)

“(...) Tivemos (capacitação sobre a vacina contra HPV) em Valença, com a coordenadora da imunização. E depois fizemos orientações nas escolas sobre qual vacina era esta e como estaria atuando." (E7)

"Fizemos capacitação com a coordenadora da imunização baseada nas normas do Ministério da Saúde." (E10)

"Fui à uma capacitação com a responsável pela rede de frios de composição, abrangência armazenamento da vacina." (E12)

As vacinas anti-HPV têm-se mostrado seguras, altamente imunogências e eficazes na prevenção da infecção HPV e lesões associadas ${ }^{19}$.

A profilaxia contra o vírus do HPV, a partir da vacinação, tem o objetivo de impedir a infecção por esse vírus, ao passo que as vacinas terapêuticas tem o objetivo de tratar a infecção já estabelecida ou a própria lesão causada pelo $\mathrm{HPV}^{20}$. As vacinas profiláticas começaram à ser pesquisadas há alguns anos nos EUA e, até o momento, têm se mostrado seguras, bem toleradas, altamente imunogênicas e eficazes contra os tipos de HPV que causam as verrugas genitais e $70 \%$ dos casos de câncer do colo de útero ${ }^{20}$.

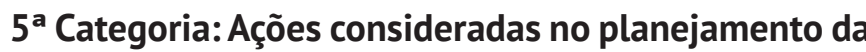 prevenção}

O planejamento das atividades de prevenção do câncer de colo de útero deve ser realizado visando à demanda, de acordo com as características da comunidade. Para isso, as particularidades de cada local, o envolvimento da comunidade, os profissionais da saúde, movimentos de mulheres e meios de comunicação devem ser considerados para uma melhor eficácia no desenvolvimento das atividades planejadas ${ }^{1}$.
"Fazemos orientações ao grupo de risco, que são as mulheres nas idades determinadas, e a coleta do exame Papanicolau também." (E4)

"São feitas reuniões de equipe, então planejamos as ações, a sala de espera, a coleta do exame preventivo uma vez por semana, entre outros." (E5)

“Campanhas (palestras), coleta do exame citopatológico, busca ativa das mulheres, garantir o tratamento do resultado,fazem parte do planejamento das ações de prevenção." (E6)

“Educação em saúde, sala de espera, busca ativa e coleta do exame citopatológico, são planejadas durante o ano." (E10)

As mulheres são estimuladas à realizarem o exame preventivo quando há busca ativa, para que sejam alcançados os índices satisfatórios de promoção da saúde são essenciais que sejam realizadas ações educativas em grupos, planejamento de ações e a posterior avaliação dos resultados ${ }^{21}$. Para isso, o profissional precisa ser capacitado para atingir de forma clara o publico alvo, a fim de acompanhar estes pacientes com segurança e conhecimento técnico-científico ${ }^{22}$.

Com a análise dos dados, foi possível verificar que existe certo conhecimento dos enfermeiros acerca da prevenção do câncer de colo de útero, mais ainda, se faz necessário uma abordagem mais qualificada sobre a temática, ressaltando a importância das ações de enfermagem na prevenção da doença.

Visto que os enfermeiros da Atenção Básica conhecem as medidas de prevenção preconizadas pelo Ministério da Saúde, como exame citopatológico de 25 a 64 anos, vacinação contra o HPV em meninas de 11 a 13 anos, e educação em saúde para população e equipe, os enfermeiros em sua maioria focam muito o exame citopatológico, deixando um pouco de lado a importância do uso de preservativo, que é a principal forma de proteção da doença.

\section{CONCLUSÃO}

É necessário conscientizar as mulheres de todas as faixas etárias, quanto às medidas de prevenção que vão desde a educação sexual, com uso de preservativo, a vacinação contra HPV e ao exame para prevenir o câncer do colo do útero periódico, medidas estas que devem estar ao alcance da população feminina de acordo com as políticas nacionais de saúde instituídas no país.

De acordo com o estudo, espera-se que possa haver uma ampliação na compreensão sobre a prevenção do câncer de colo de útero e a importância da atuação do enfermeiro, contribuindo na redução de surgimento de novos casos, podendo melhorar a qualidade no atendimento às mulheres nas unidades de Atenção Básica no município.

0 estudo aponta que deve haver planejamento direcionado para projetos educativos para a prevenção 
do câncer do colo de útero, destacando sua importância e seus objetivos, bem como maior atenção por parte dos gestores, não deixando que falte material para realização do exame citopatológico, estruturar as unidades tanto no que diz respeito a estrutura física adequada, materiais e um quantitativo de profissionais de saúde qualificados para atender todo município, permitindo um melhor atendimento ao público, sobre a prevenção do câncer do colo do útero, conforme as recomendações do Ministério da Saúde.

\section{REFERÊNCIAS}

1. Melo MCSC, Vilela F, Salimena AMO, Souza IEO. O Enfermeiro na Prevenção do Câncer do colo de Útero: o Cotidiano da Atenção Primária. Revista Brasileira de Cancerologia. [Internet] 2012 jul. [citado 2015 out 24]; 58(3): 389-398. Disponível em: http://www1.inca.gov.br/rbc/n_58/v03/ pdf/08_artigo_enfermeiro_prevencao_cancer_colo_utero_ cotidiano_atencao_primaria.pdf.

2. Brasil. Ministério da saúde. Estimativa 2014: Incidência de Câncer no Brasil. Rio de Janeiro: INCA [Internet]. 2014 [citado 2015 jul. 08]. Disponível em: http://www.inca.gov.br/ estimativa/2014/estimativa-24042014.pdf.

3. Melo SCCS, Prates L, Carvalho MDB, Marcon SS, Pelloso SM. Alterações citopatológicas e fatores de risco para a ocorrência do câncer de colo uterino. Rev. Gaúcha Enferm. [Internet]. 2009 [citado 2015 ago 29]; 30 (4): 602- 608. Disponível em http:// www.scielo.br/scielo.php?script=sci_arttext \& pid=S198314472009000400004\&lng=en. http://dx.doi.org/10.1590/ S1983-14472009000400004.

4. Figueiredo NMA, Leite JL. et al. Enfermagem Oncológica Conceitos e Práticas. São Caetano do Sul: Yendis; 2009.

5. Ferreira MLSM, Galvão MTG. Avaliação do risco de câncer de colo uterino em trabalhadoras da indústria têxtil. Cienc Cuid Saude [Internet]. 2009 [citado 2015 ago 29]; 8(1):8692. Disponível em http://periodicos.uem.br/ojs/index.php/ CiencCuidSaude/article/viewFile/7780/4413.

6. Brasil. Ministério da Saúde. Ações de Enfermagem para o Controle do Câncer: Uma proposta de integração ensinoserviço. Rio de Janeiro: INCA [Internet]. 2008 [citado 2014 jul. 08]. Disponível em: http://www.inca.gov.br/enfermagem/.

7. Brasil. Ministério da saúde. Brasil. Vacinação contra HPV começa nesta segunda-feira (10). Portal Brasil [Internet]. 2014 [citado 2015 ago. 17]. Disponível em: http://www.brasil. gov.br/saude/2014/03/vacinacao-contra-hpv-comeca-nestasegunda-feira-10.

8. Casarin MR, Piccoli JCE. Educação em saúde para prevenção do câncer de colo do útero em mulheres do município de Santo Ângelo/RS. Ciênc. saúde coletiva. [Internet]. 2011 [citado 2015 ago 17]; 16 (9): 3925-3932. Disponível em: http://www.scielo.br/scielo. php?pid=S1413-81232011001000029\&script=sci_arttext.

9. Sales LVMC. A Atuação do enfermeiro na prevenção do câncer de colo uterino [monografia]. Belo Horizonte: Faculdade de Medicina da Universidade Federal de Minas Gerais; 2012.

10. Mistura C, Mistura C; Silva RCCS, et al. Papel do Enfermeiro na Prevenção do Câncer de Colo Uterino na Estratégia Saúde da Família. Revista Contexto Saúde [Internet]. 2011 [citado 2015 nov. 07]; 10 (20): 1161 - 1164. Disponível em: https://www. revistas.unijui.edu.br/index.php/contextoesaude/article/ view/1763/1467.

11. Frigato $S$, Hoga LAK. Assistência à mulher com câncer de colo uterino: o papel da enfermagem. Revista Brasileira de Cancerologia [Internet]. 2003 [citado 2015 nov. 07]; 49(4): 209-214. Disponível em http://www.inca.gov.br/rbc/n_49/ v04/pdf/ARTIG01.pdf.

12. Minayo MCS. O desafio do conhecimento. Pesquisa qualitativa em saúde. $10^{a}$ edição revista e aprimorada. São Paulo: Hucitec; 2010.

13. Instituto Brasileiro de Geografia e Estatística (IBGE). Estimativas populacionais dos municípios em 2010 [Internet]. 2010 [citado 2015 nov. 08]. Disponível em: http://cidades. ibge.gov.br/xtras/perfil.php?codmun=330610.

14. Bardin L. Análise de Conteúdo. Lisboa, Portugal; Edições 70, LDA, 2009.

15. Domingues TAM, Chavez EC. O conhecimento científico como valor no agir do enfermeiro. Rev Esc Enferm USP.[Internet]. 2005 [citado 2015 nov. 07]; 39 (Esp.): 580-588. Disponível em: http://www.ee.usp.br/reeusp/upload/pdf/82.pdf.

16. Beghini AB, Salimena AMO, Melo MCSC, Souza IEO. Adesão das AcadêmicasdeenfermagemàPrevençãodoCâncerGinecológico: da Teoria à Prática. Texto contexto - enferm. [Internet]. 2006 [citado 2015 out. 20]; 15 (4): 637-644. Disponível em: http:// www.scielo.br/scielo.php?script=sci_arttext \& pid=S010407072006000400012\&lng=en. http://dx.doi.org/10.1590/ S0104-07072006000400012.

17. Souza GG. A importância de ações educativas para prevenção do câncer de colo uterino no contexto da Estratégia Saúde da Família. [Trabalho de Conclusão de Curso de Especialização]. Belo Horizonte: Universidade Federal de Minas Gerais; 2012.

18. Siqueira GS, et al. Citopatologia como prevenção do câncer do colo uterino. Cadernos de Graduação - Ciências Biológicas e da Saúde - UNIT [Internet]. 2014 [citado 2015 nov. 07]; 2(1):37-49Disponível em: https://periodicos.set.edu.br/index. php/cadernobiologicas/article/view/1179/740.

19. Giraldo PC, et al. Prevenção da infecção por HPV e lesões associadas com o uso de vacinas. DST - Jornal Brasileiro de Doenças Sexualmente Transmissíveis [Internet]. 2008 [citado 2015 out. 29]; 20 (2):132-140, Disponível em: http://www.hu.ufsc.br/projeto_hpv/PREVENCAO\%20 DA\%20INFECCAO\%20POR\%20HPV\%20E\%20LESOES\%20 ASSOCIADAS.pdf.

20. Bragueto T, Suzuki LE. Vacinas contra o Papilomavírus Humano - HPV. Newslab [Internet]. 2008 [citado 2015 out. 29]; 87: 58-68. Disponível em: http://www.newslab.com.br/ ed_anteriores/87/art03.pdf.

21. Marçal JÁ, Gomes LTS. A prevenção do câncer de colo de útero realizada pelo enfermeiro na Estratégia Saúde da Família: Revisão integrativa da literatura. Revista Eletrônica Acervo Saúde [Internet]. 2013 [citado 2015 nov. 07]; 5(2): 474-489. Disponível em: http://acervosaud.dominiotemporario.com/ doc/artigo_035.pdf.

22. Tomio SJD. Perfil dos pacientes cadastrados no serviço de assistência ao portador de feridas no sistema único de saúde de Itajaí. Anais do V Congresso Brasileiro de Prevenção e Tratamento de Feridas. Revista Enfermagem Atual In Derme [Internet]. 2015 [citado 2017 abr 01]; 75. Disponível em http://inderme.com.br/revistas/revista_13.pdf. 